\title{
New physics searches at a Linear Collider with polarized beams
}

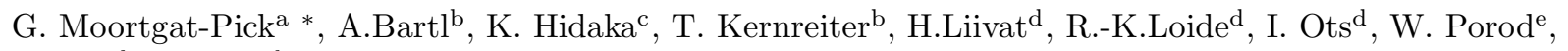 \\ R.Saar ${ }^{\text {d }}$ H. Uibo ${ }^{\mathrm{d}}$

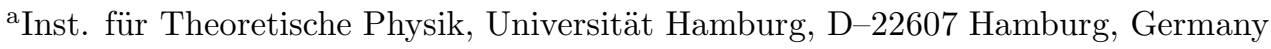 \\ ${ }^{b}$ Institut für Theoretische Physik, Universität Wien, A-1090 Vienna, Austria \\ ${ }^{\mathrm{c}}$ Dept. of Physics, Tokyo Gakugei University, Koganei, Tokyo 184-8501, Japan \\ dinstitute of Physics, University of Tartu, Riia 142, 51014 Tartu, Estonia \\ ${ }^{\mathrm{e}}$ Inst. für Theor. Physik, Universität Zürich, CH-8057 Zürich, Switzerland
}

\begin{abstract}
A future $e^{+} e^{-}$Linear Collider has a large physics potential for the discovery of new physics beyond the Standard Model and precision studies of the Standard Model itself. It is well suited to complement and extend the physics program of the LHC concerning the precise determination of the underlying theory. The use of polarized beams at a $\mathrm{LC}$ will be one of the decisive tools.
\end{abstract}

\section{Introduction}

A future Linear Collider (LC) designed with high luminosity of about $\mathcal{L}=3.4 \cdot 10^{34} \mathrm{~cm}^{-2} \mathrm{~s}^{-1}$ at $\sqrt{s}=500 \mathrm{GeV}$ and $\mathcal{L}=5.8 \cdot 10^{34} \mathrm{~cm}^{-2} \mathrm{~s}^{-1}$ at $\sqrt{s}=800 \mathrm{GeV}$ will provide precision studies in the energy range between LEP and the TeV range [1. Concerning the searches for New Physics it complements and extends the physics program of the Hadron Colliders, Tevatron and the future Large Hadron Collider (LHC)円. The LC has large potential for the discovery of new particles and is unique concerning the precision analysis of new physics (NP) as well as of the Standard Model (SM) [1]. An important tool of a LC is the use of polarized beams [2]. Already in the base line design it is foreseen to use the electron beams polarized to around $80 \%$ via a strained photocathode technology. In order to generate also polarized positrons the use of a helical undulator is favoured, producing polarized photons which generate via pair production polarized positrons of about $40 \%$ (with full intensity of the $e^{+}$beam) to $60 \%$ (with $55 \%$ intensity).

In the next sections we summarize the new physics searches at a LC starting from high precision studies of the SM as a motivation.

\footnotetext{
*Talk given by G. Moortgat-Pick, gudrid@mail.desy.de

${ }^{1}$ contact Georg Weiglein, see http://www.ippp.dur.ac.uk/ q georg/lhclc/
}

\section{High precision analysis of the SM}

One possibility to see hints for new physics, even if new particles are not yet found, are electroweak precision tests with an unprecedented accuracy.

2.1. Triple gauge couplings in $e^{+} e^{-} \rightarrow Z \gamma$

The study of triple gauge couplings is very promising. In [3] a method has been worked out to derive complete analytical expressions for the spin polarization and alignment of the $Z$ boson in $e^{+} e^{-} \rightarrow Z \gamma$ including the contributions from $Z Z \gamma$ and $Z \gamma \gamma$ anomalous couplings.

2.2. Anomalous couplings in $e^{+} e^{-} \rightarrow W^{+} W^{-}$ Searches for deviations from the SM have already been studied, comparing polarized cross sections in a general parametrization including anomalous gauge couplings with expected precision measurements at the LC [1]. It turns out that for this purpose the polarization of the beams is very powerful: e.g. the polarization of $P\left(e^{-}\right)= \pm 80 \%$ (together with $P\left(e^{+}\right)=\mp 60 \%$ ) improves the sensitivity up to a factor 1.8 (2.5).

\subsection{Transversely polarized beams in} $e^{+} e^{-} \rightarrow W^{+} W^{-}$

A promising possibility to study electroweak symmetry breaking is the use of transversely po- 
larized $e^{+} e^{-}$beams which projects out $W_{L}^{+} W_{L}^{-}$ [4]. The asymmetry with respect to the azimuthal angle of this process focusses on the $L L$ mode. This asymmetry is very pronounced at high energies reaching about $10 \%$. The advantage of this observable is that at high energies this asymmetry peaks at larger angles and not in beam direction where the analysis might be difficult. One has to note, however, that for the use of transverse beams the polarization of both beams is needed. The effect does not occur if only one beam is polarized since the cross section is given by:

$$
\begin{aligned}
\sigma= & \left(1-P_{e^{-}}^{L} P_{e^{+}}^{L}\right) \sigma_{u n p}+\left(P_{e^{-}}^{L}-P_{e^{+}}^{L}\right) \sigma_{p o l}^{L} \\
& +P_{e^{-}}^{T} P_{e^{+}}^{T} \sigma_{p o l}^{T} .
\end{aligned}
$$

\subsection{GigaZ}

A very spectacular method for probing the SM is foreseen as an upgrade of a LC: the GigaZ option. Here $e^{+} e^{-} \rightarrow Z \rightarrow f \bar{f}$ is studied at the pole with very high luminosity of about $10^{9}$ Z's within three month. With this option the effective electroweak leptonic mixing angle can be measured via the left-right asymmetry of this process with an unprecedented accuracy, see Table 1 [1].

\subsection{Sensitivity to $\mathrm{CP}$ violation in the $\mathrm{SM}$}

In [6] electroweak dipole form factors of the $\tau$ lepton have been analysed with regard to $\mathrm{CP}$ violation. $\mathrm{CP}-$ odd triple product correlations have been studied and sensitivity bounds for the real and imaginary parts of these form factors have been set. Using polarized beams a LC could be sensitive to these form factors up to $O\left(10^{-19}\right)$ ecm.

\section{Revealing the structure of Susy}

Supersymmetry is widely regarded as the best motivated extension of the SM. However, since the SM particles and their Susy partners are not mass degenerate, Susy has to be broken, which leads even for its minimal version, the Minimal Supersymmetric Standard Model (MSSM), to about 100 free parameters. In specific scenarios of Susy breaking one has much less parameters: 5 in mSUGRA, 4 in AMSB and 5 in GMSB.

In order to exactly pin down the structure of the underlying model it is unavoidable to extract the parameters without assuming a partic- ular breaking scheme. The LC with its clear signatures is well suited to determine the general parameters with high precision and to test fundamental Susy assumptions as e.g. the equality of quantum numbers or of couplings of the particles and their Susy partners. It turns out that the use of polarized beams plays a decisive role in this context.

\subsection{Stop mixing angle in $e^{+} e^{-} \rightarrow \tilde{t}_{1} \tilde{t}_{1}$}

As demonstrated in [1] the mass and the mixing angle of $\tilde{t}$ can be extracted with high precision via the study of polarized cross sections for light stop production. At a high luminosity LC and with $P\left(e^{-}\right)=80 \%$ and $P\left(e^{+}\right)=60 \%$ an accuracy of $\delta\left(m_{\tilde{t}_{1}}\right) \approx 0.8 \mathrm{GeV}$ and $\delta \cos \theta_{\tilde{t}} \approx 0.008$ could be reachable.

\subsection{Quantum numbers in $e^{+} e^{-} \rightarrow \tilde{e}_{L, R}^{+} \tilde{e}_{L, R}^{-}$}

Susy transformations associate $e_{L, R}^{-} \leftrightarrow \tilde{e}_{L, R}^{-}$ and the antiparticles $e_{L, R}^{+} \leftrightarrow \tilde{e}_{R, L}^{+}$. In order to prove this association of scalar particles to chiral quantum numbers the use of polarized beams is necessary [7. The process occurs via $\gamma$ and $Z$ exchange in the $\mathrm{s}-$ channel and via $\tilde{\chi}_{i}^{0}$ exchange in the $\mathrm{t}$-channel. Only in the $\mathrm{t}$-channel the SM particle is directly coupled to its scalar partner and in order to test the association of quantum numbers one has to projects out the $\mathrm{t}-$ channel exchange.

With completely polarized $e_{L}^{-} e_{L}^{+}$only $\tilde{e}_{L}^{-} \tilde{e}_{R}^{+}$ contributes. Due to their $L, R$ coupling character $\tilde{e}_{L}, \tilde{e}_{R}$ can be discriminated via their decays and can be identified via their charge. One has to note that a polarized $e^{+}$beam is necessary. Even completely polarized $e^{-}$would not be sufficient, since otherwise the s-channel exchange could not be switched off. In reality partially polarized beams of $P_{e^{-}}=-80 \%$ and $P_{e^{+}}=-60 \%$ can be sufficient to probe this association. With this polarization $\tilde{e}_{L}^{-} \tilde{e}_{R}^{+}$dominates e.g. by a factor of 3, Fig. 11.

\subsection{Gaugino/higgsino sector}

In 8] and references therein a strategy has been worked out to determine accurately the MSSM parameters $M_{1}, \Phi_{M_{1}}, M_{2}, \mu, \Phi_{\mu}$ and moderate $\tan \beta$ via the study of polarized rates in $e^{+} e^{-} \rightarrow \tilde{\chi}_{i}^{ \pm} \tilde{\chi}_{j}^{\mp}$ and $e^{+} e^{-} \rightarrow \tilde{\chi}_{i}^{0} \tilde{\chi}_{j}^{0}$. Even if only 
$\tilde{\chi}_{1}^{+} \tilde{\chi}_{1}^{-}, \tilde{\chi}_{1}^{0} \tilde{\chi}_{2}^{0}$ were accessible, it would be sufficient for determining the MSSM parameters [8]. With light chargino pair production and polarized initial beams one can unambiguously determine the mixing angle but $M_{2}, \mu, \tan \beta$ still depend on $m_{\tilde{\chi}_{2}^{ \pm}}$. If one furthermore includes now the light neutralino system and studies the polarized cross sections $e^{+} e^{-} \rightarrow \tilde{\chi}_{1}^{0} \tilde{\chi}_{2}^{0}$ in the complex $M_{1}$ plane, one can simultaneously determine the parameter $M_{1}$ and predict $m_{\tilde{\chi}_{2}^{ \pm}}$.

Once the parameters are determined one can efficiently test whether the gauge couplings $g_{B e e}$ and $g_{W e e}$ are identical to the Yukawa couplings $g_{\tilde{B} e \tilde{e}}$ and $g_{\tilde{W} e \tilde{e}}$, respectively, by studying the polarized cross sections with a variable ratio of $g_{B e e} / g_{\tilde{B} e \tilde{e}}$ and $g_{W e e} / g_{\tilde{W} e \tilde{e}}$ and comparing it with experimental values [8].

\subsection{Case of high $\tan \beta: \tau$ polarization}

In case of high $\tan \beta>10$ the chargino and neutralino sector is insensitive to this parameter. However, one could then determine $\tan \beta$ from another sector whose particles are relatively light in many scenarios: the $\tilde{\tau}$ sector.

The polarization of $\tau$ 's from $\tilde{\tau}_{i} \rightarrow \tau \tilde{\chi}_{1}^{0}$ is sensitive to $\tan \beta$. In case of a sufficient higgsino admixture in the $\tilde{\chi}_{1}^{0}$ it is even possible to determine high $\tan \beta$ as well as $A_{\tau}$, without any assumptions on the Susy breaking mechanism [9]: after determining the $\tilde{\tau}$ mixing angle via the polarization asymmetry $A_{\text {pol }}=\left(\sigma_{L}-\sigma_{R}\right) /\left(\sigma_{L}+\sigma_{R}\right)$ in $e^{+} e^{-} \rightarrow \tilde{\tau}_{1} \tilde{\tau}_{1}$, one can determine $\tan \beta$ from the $\tau$ polarization in the decay $\tilde{\tau} \rightarrow \tau \tilde{\chi}_{1}^{0}$, see Fig.2. Even for high $\tan \beta \approx 40$ one can reach an accuracy of about $10 \%$. Measuring now $m_{\tilde{\tau}_{2}}$ leads to the determination of $A_{\tau}$.

\section{5. $\mathbf{C P}-$ phases in the $\tilde{\tau}$ sector}

In the $\tilde{\tau}_{i}$ system $\mathrm{CP}$-violating phases can occur in the parameters $A_{\tau}, \mu$ and $M_{1}$. These phases have a decisive influence on masses, couplings and various $\mathrm{CP}$-violating observables. The influence of the phases could be quite strong even on $\mathrm{CP}$-conserving decay branching ratios (BR's) and hence one should take it into account when determining the MSSM parameters at the LHC and LC. In this context the BR's also might be a sensitive probe for the $\mathrm{CP}-$ phases 10 .

\subsection{Extended Susy models}

In case of R-parity violating Susy nonstandard couplings could occur which produce a scalar particle in the s-channel: $e^{+} e^{-} \rightarrow \tilde{\nu} \rightarrow$ $e^{+} e^{-}$. The process gives a significant signal over the background. Since it requires both lefthanded $e^{-}$and $e^{+}$beams, it can be easily analysed and identified by the use of beam polarization ([2] and references therein): here simultaneously polarized beams enhance the signal by about a factor of 10 .

Introducing in the MSSM an additional Higgs singlet leads to the $(\mathrm{M}+1) \mathrm{SSM}$ with one additional neutralino. Since the mass spectra of the four light neutralinos could be similar to those in the MSSM a distinction between the models might be difficult via spectra and rates alone. However, polarization effects might then indicate the different coupling structure in the $(\mathrm{M}+1) \mathrm{SSM}$ [11].

\section{Other kind of New Physics}

Another approach to resolve the hierachy problem is the introduction of large extra dimensions. At a $\mathrm{LC}$ the process $e^{+} e^{-} \rightarrow \gamma G$ is promising and it has been worked out that running on two different $\sqrt{s}$ one can determine the number of extra dimensions [1]. The use of polarized beams in this context enlarges on one hand the sensitivity to the new scale $M_{*}$ and suppresses on the other hand the main background $e^{+} e^{-} \rightarrow \nu \nu \gamma$ significantly. The ratio $S / \sqrt{B}$ is enhanced by a factor of about 2.1 (4.4) if $P\left(e^{-}\right)=+80 \%$ (and $\left.P\left(e^{+}\right)=-60 \%\right)$ is used.

\section{Summary}

It has been shown that a $\mathrm{LC}$ in the $\mathrm{TeV}$ range is well suited for new physics searches and in particular for its precise determination. The use of polarized beams plays a decisive role円. In this context the use of simultaneously polarized $e^{-}, e^{+}$beams has several advantages: enhancing considerably the accuracy for SM precision tests at GigaZ, providing higher sensitivity to nonstandard couplings and supplying efficient tools to identify quantum numbers of the new particles

${ }^{2}$ for updates see POWER group (POlarization at Work in Energetic Reactions), forum for machine physicists, experimentalists, theorists: http://www.desy.de/ gudrid/POWER/ 
Table 1

Ew. precision parameters at different colliders [5]

\begin{tabular}{l|cccc} 
Accuracy & LEP/Tev. & Tev./LHC & LC & GigaZ \\
\hline$\delta M_{W}$ & $34 \mathrm{MeV}$ & $15 \mathrm{MeV}$ & $15 \mathrm{MeV}$ & $6 \mathrm{MeV}$ \\
$\delta \sin ^{2} \theta_{\text {eff }}$ & 0.00017 & 0.00017 & 0.00017 & 0.00001 \\
$\delta m_{t}$ & $5 \mathrm{GeV}$ & $2 \mathrm{GeV}$ & $0.2 \mathrm{GeV}$ & $0.2 \mathrm{GeV}$ \\
$\delta m_{h}$ & - & $0.2 \mathrm{GeV}$ & $0.05 \mathrm{GeV}$ & $0.05 \mathrm{GeV}$
\end{tabular}

and to determine the underlying parameters of the theory.

G.M.-P. was partially supported by the Graduiertenkolleg 'Zukünftige Entwicklungen in der Teilchenphysik' of the University of Hamburg, Project No. GRK 602/1. This work was also supported by the EU TMR Network Contract No. HPRN-CT-2000-00149. The group from IP, University of Tartu was partly supported by ESF grant 4510.

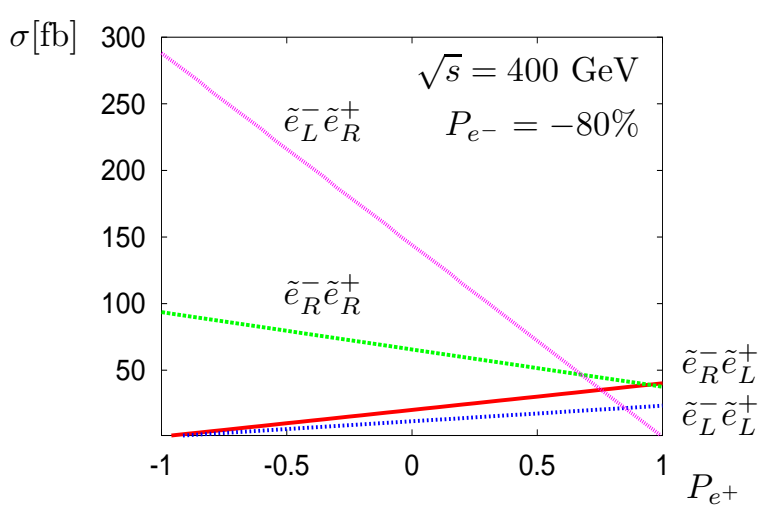

Figure 1. Test of selectron quantum numbers in $e^{+} e^{-} \rightarrow \tilde{e}_{L, R}^{+} \tilde{e}_{L, R}^{-}$with fixed electron polarization $P\left(e^{-}\right)=-80 \%$ and variable positron polarization $P\left(e^{+}\right)$. For $P\left(e^{-}\right)=-80 \%$ and $P\left(e^{+}\right)<0$ both pairs $\tilde{e}_{L}^{-} \tilde{e}_{R}^{+}$and $\tilde{e}_{R}^{-} \tilde{e}_{R}^{+}$still contribute. For $P\left(e^{+}\right)=-60 \% \tilde{e}_{L}^{-} \tilde{e}_{R}^{+}$dominates by more than a factor 3 [7].

\section{REFERENCES}

1. J. A. Aguilar-Saavedra et al., ECFA/DESY LC Physics Working Group Collaboration, hep-ph/0106315.

2. G. Moortgat-Pick and H. M. Steiner, Eur. Phys. J. directC 6 (2001) 1 hep-ph/0106155.

3. I. Ots, H. Uibo, H. Liivat, R. K. Loide and R. Saar, Nucl. Phys. B 588 (2000) 90 and Possible $Z \gamma \gamma$ and $Z Z \gamma$ coupling in $e^{+} e^{-} \rightarrow$

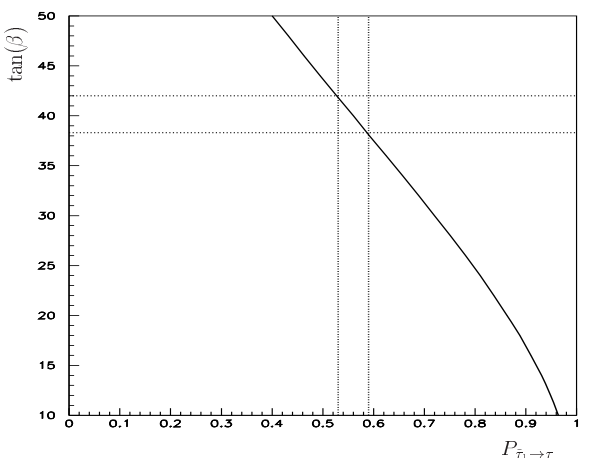

Figure 2. Measuring the $\tau$ polarization $P_{\tilde{\tau}_{1} \rightarrow \tau}$ from the decay $\tilde{\tau}_{1} \rightarrow \tau \tilde{\chi}_{1}^{0}$ for an already measured mixing angle $\cos \theta_{\tilde{\tau}}$ leads in an example of [9] to an accurate determination of high $\tan \beta: \tan \beta=$ $40 \pm 2$.

$Z \gamma$ in a general relativistic density matrix formalism, Contrib. to ICHEP02, Amsterdam.

4. J. Fleischer, K. Kolodziej and F. Jegerlehner, Phys. Rev. D 49 (1994) 2174.

5. J. Erler, S. Heinemeyer, W. Hollik, G. Weiglein and P. M. Zerwas, Phys. Lett. B 486 (2000) 125.

6. B. Ananthanarayan, S. D. Rindani and A. Stahl, hep-ph/0204233.

7. C. Blöchinger, H. Fraas, G. Moortgat-Pick and W. Porod, Eur. Phys. J. C 24 (2002) 297.

8. S. Y. Choi, J. Kalinowski, G. Moortgat-Pick and P. M. Zerwas, Eur. Phys. J. C 22 (2001) 563 ; S. Y. Choi, J. Kalinowski, G. MoortgatPick and P. M. Zerwas, Eur. Phys. J. C 23 (2002) 769 hep-ph/0202039.

9. E. Boos, U. Martyn, G. Moortgat-Pick, M. Sachwitz, S. Vologdin, P.M. Zerwas, in prep..

10. A. Bartl, K. Hidaka, T. Kernreiter and W. Porod, hep-ph/0207186 and Phys. Lett. B 538 (2002) 137.

11. S. Hesselbach, F. Franke, H. Fraas, hepph/0003272; F. Franke and S. Hesselbach, Phys. Lett. B 526 (2002) 370. 